\title{
Eine neue Netzgarnitur mit Kipp-Schliessmechanismus für quantitative Zooplanktonfänge in Seen
}

\begin{abstract}
ABSTRAC'T
A new net with tilt-closing mechanism for quantitative zooplankton sampling in lakes A new set of nets in twin arrangement for quantitative layer-sampling of crustaceans is presented and compared to a volume sampler, as regards capturing characteristics. Due to a favorable geometry and clearly defined opening of the net, there is no loss due to flight reaction nor water backup.
\end{abstract}

Für trophodynamische Betrachtungen von Planktonorganismen grösserer Gewässer sind quantitative Zooplanktonfänge unerlässlich. In Produktionsmodellen wird dabei oft auch die Entwicklungsdauer der Organismen benötigt. Da diese aber von der Temperatur abhängt, muss auch bekannt sein, wie lange sich die einzelnen Tierarten im Laufe ihrer täglichen Migration in verschieden temperierten Wasserschichten aufhalten. Dies setzt eine Probenahmeeinrichtung voraus, welche quantitativ und störungsfrei arbeitet (Nachtprobenahmen) und zudem schnell ist. Volumenproben mit geeigneten Wasserschöpfern [4,6] oder Pumpfänge [5] liefern zwar gute quantitative Resultate, wenn sie der Fluchtreaktion der schnelleren Crustaceen zuvorkommen [2, 3], sind aber in tieferen Seen zu wenig integrierend und relativ langsam. Stufenfänge mit den üblichen Nansen-Schliessnetzen liefern demgegenüber vertikal integrierte Proben, welche aber quantitativ schlecht auswertbar sind, weil je nach Verstopfungsgrad durch Algen usw. ungleich viel Wasser filtriert wird. Zudem ist die Eintrittsöffnung bei den niederen Zuggeschwindigkeiten, wie sie beim vertikalen Fang angewendet werden, nicht definiert. Das Verhältnis von Eintrittsöffnung zu filtrierender Netzfläche liegt oft im Bereich von 1:10 bis 1:20. Ein weiterer Nachteil der Netzmethode besteht darin, dass Leine und Schliessmechanismus vor der Netzöffnung hergezogen werden und eine Fluchtreaktion senkrecht zur Netzachse auslösen.

Bei quantitativen Planktonfängen darf das Netz nicht gestoppt werden, bevor es geschlossen ist, andernfalls kann ein Teil der gefangenen Tiere wieder entweichen. Wird einer der üblichen, durch ein Fallgewicht zu aktivierenden Schliessmechanismen verwendet, so muss die Laufzeit des Fallgewichtes berücksichtigt werden, was erfahrungsgemäss zu Ungenauigkeiten führt.

Durch die Zwillingsanordnung unserer Netze kann die Aufhängung mit dem Schliessmechanismus von den Eintrittsöffnungen weg verlagert werden. Diese sind durch einen Metallkragen von $12 \mathrm{~cm}$ Innendurchmesser klar definiert. Das Netz wird mittels einer eingezogenen Kordel in einer Nut am Metallring befestigt (und kann auch leicht ausgetauscht werden). Durch stromlinienförmige Form aus 
dichtem Gewebe im oberen Teil und dem eigentlichen Netz aus Hochleistungsgewebe im unteren Teil ergibt sich eine Vergrösserung der Netzfläche. Das Verhältnis von Eintrittsöffnung zu Netzfläche verbessert sich dadurch auf 1:48. Eine Verstopfung durch Algen ist damit auch bei längeren Fangstrecken vernachlässigbar. Die Maschenweite basiert auf der Grösse der kleinsten Crustaceenstadien (im Greifensee sind dies Nauplien mit etwa $100 \mu \mathrm{m}$ Abmessung). Pro Netz wurden $0,5 \mathrm{~m}^{2}$ monofiles Nylongewebe der Bezeichnung 14 P-95 der Firma «Schweizerische Seidengazefabrik AG», Zürich, mit $95 \mu \mathrm{m}$ Maschenweite und $43 \%$ offener Fläche verarbeitet. Aus der gleichen Quelle sind heute Maschenweiten zwischen $1 \mu \mathrm{m}$ und $4 \mathrm{~mm}$ in feinen Abstufungen erhältlich.

Um das Netz rasch eintauchen und absenken zu können, wurde der Trichter aus massivem Messing gefertigt. Dank dem relativ grossen Abstand der beiden Netze $(60 \mathrm{~cm})$ sind Verwicklungen praktisch ausgeschlossen. Die Kombination der Netzgarnitur mit der Schliessvorrichtung nach Ambühl [1] erlaubt ein verzögerungsfreies Schliessen der Netze durch Umkippen des Metallkragens um etwa $150^{\circ}$ nach unten (Abbildung). Der obere Kegelstumpf aus dichtem TETEX-Gewebe verschliesst nun das Netz.
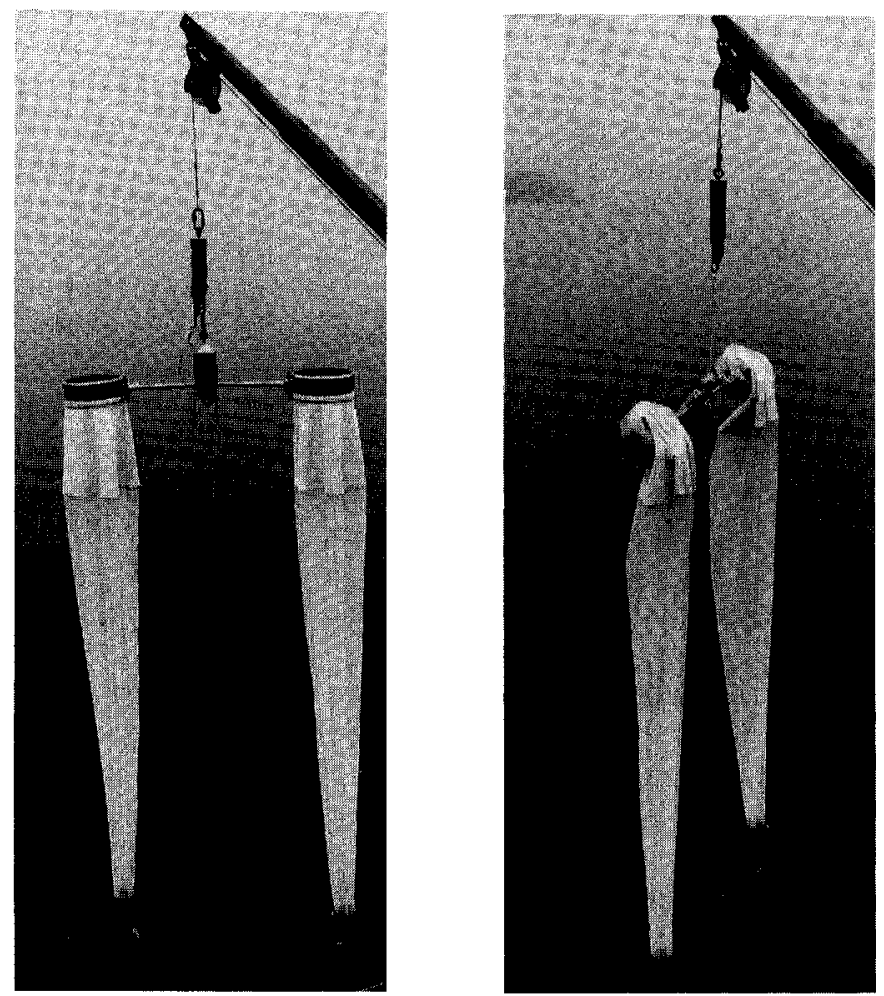

Schliessnetz mit Fernauslöser in geöffnetem und geschlossenem Zustand. Tilt-closing net with remote releaser, open and closed. 
Um genügend genaue Resultate für statistische Zwecke zu erhalten, wären 5 und mehr Netzzüge erforderlich. Dies ist aber in der Praxis sehr oft unerfüllbar, weil bei diurnalen Serien mit mehreren Tiefenstufen die Zeit für eine horizontale Integration nicht ausreicht (sofern nicht mit mehreren Garnituren synchron gearbeitet wird). Mit der Zwillingsnetzgarnitur wird immerhin die kleinräumige Patchiness ausgeglichen. Vergleich der Fangkapazität der Netzgarnitur mit dem grossvolumigen Wasserschöpfer nach Ambühl [1]
in der Tiefenstufe 0-30 m des Vierwaldstättersees am 28. Juli 1982.

\begin{tabular}{|c|c|c|}
\hline Gattung/Art & $\begin{array}{l}\text { Netzgarnitur } \\
\text { Ind. } / \mathrm{m}^{2}\end{array}$ & $\begin{array}{l}\text { Volumenprobe } \\
\text { Ind. } / \mathrm{m}^{2}\end{array}$ \\
\hline Daphnia spp.juvenil & 61000 & 64000 \\
\hline Daphnia spp. adult & 25000 & 22000 \\
\hline Eudiaptomus gracilis juvenil & 149000 & 160000 \\
\hline Eudiaptomus gracilis adult & 49000 & 18000 \\
\hline Cyclops spp., $\mathrm{C}_{1}-\mathrm{C}_{5}$ & 88000 & 57000 \\
\hline Cyclops spp. adult & 27000 & 30000 \\
\hline
\end{tabular}

Der Vergleich von volumetrischen Sofortfängen ( 7 Tiefen) mit den Netzzügen (6 Stufen) zeigt keine signifikanten Unterschiede der beiden Methoden. Insbesondere kann eine Fluchtreaktion der schnellen Copepoden ausgeschlossen werden. Die deutlichen Unterschiede bei den adulten Eudiaptomiden rühren vermutlich von ihrer ungleichmässigen Einschichtung her, welche von den Schöpfproben nicht integral zu erfassen war (Tabelle).

Bei direkter Beobachtung des Filtrationsvorgangs im Oberflächenwasser ist kein Überstau des Wassers vor dem Netz erkennbar, solange die Regel eingehalten wird, dass nicht schneller als $0,5 \mathrm{~m} / \mathrm{s}$ gezogen werden darf. Als Indikatoren einer Bewegung von der Netzöffnung weg (oder zur Netzöffnung hin) dienten dabei kleine, ins Wasser eingemischte Vogelfedern. Soweit Crustaceen von Auge sichtbar sind, zeigen sie beim Nahen des Netzes keine Fluchtreaktion, sondern werden genau ihrer Position entsprechend vom Netz erfasst oder ausgeschlossen. Damit ist auch die wichtigste Bedingung für ein quantitatives Arbeiten erfüllt.

H. R. Bürgi

Adresse des Autors: Dr. H.R. Bürgi, EAWAG, CH-8600 Dübendorf, Schweiz.

Das Gerät ist erhältlich bei Fa. Dr. H. Züllig, Apparatebau, CH-9424 Rheineck.

\section{LITERATURVERZEICHNIS}

1 Ambühl, H.: Schweiz. Z. Hydrol. 45 (2), 502 (1983).

2 Elster, H. J.: Ber. limnol. Flußst. Freudenthal $I V(1953)$.

3 Elster, H.J.: Verh. int. Verein. theor. angew. Limnol. 13, 961-973 (1958).

4 Langeland, A., und Rognerud, S.: Arch. Hydrobiol. 73/3, 403-410 (1974).

5 Langford, R. R.: J. Fish. Res. Bd Can. 10/5, 238-252 (1953).

6 Schindler, D. W.: J. Fish. Res. Bd Can. 26/7, 1948-1955 (1969). 